\title{
Application and Research on an Improved Clustering Method in Teaching Evaluation \\ Shaorong Feng
}

School of Information Science and Engineering, Xiamen University, 361005 Xiamen, China

shaorong@xmu.edu.cn

Keywords: Teaching evaluation; Weight; K-medoids clustering; Evaluation index; Teaching quality

\begin{abstract}
In order to analyze the teaching evaluation data effectively, based on the diversity of the weight of different evaluation indexes, this paper focuses on the issue of the current evaluation index weight setting, the method of combined weight distribution is proposed. In order to improve the accuracy of clustering, the weight is introduced to the nearest neighbor $K$-medoids clustering algorithm. The experimental results of UCI data set and the teaching evaluation data show that the proposed algorithm is feasible and effective in the teaching evaluation data analysis.
\end{abstract}

\section{一种改进的聚类方法在教学评价中的应用研究}

\author{
冯少荣 \\ 厦门大学 信息科学与技术学院，中国厦门 361005 \\ shaorong@xmu.edu.cn
}

摘要: 为了对教学评价数据进行有效分析, 在兼顾不同评价指标权重存在差异的基础上, 本 文提出组合权重分配方法, 解决目前评价指标权重设置不合理问题。通过在近邻 K-medoids 聚类算法中引入权重, 对其进行改进, 以便提高聚类准确率。对 UCI 数据集及教评数据的实 验结果表明, 所提算法在有效分析教评数据方面是可行的。

关键词：教学评估；权重；K-medoids 聚类; 评价指标；教学质量

\section{1. 引言}

随着信息科学技术和经济社会的发展，提高国民综合素质、改善人才培养方式已成为当今社 会关注的重点。高校是人才的发源地，培养高科技人才是当今社会推进教育事业改革的主题, 所以对于教学质量的提高, 高校有着义不容辞的责任和义务, 如何在教学过程中提高教学质 量成为当今高校关注的重中之重[1], 这就需要建立一个科学的评教体系对教学质量过程中的 现状进行统计和分析。

教学评价[2]是在教学目标的基础上，参照科学的标准，采用有效的技术手段，测量教学过程 和结果, 并进行价值判断的过程。教学评价一般包括教师素质、教学过程、教学内容、教学 方法、教学效果、教学管理等评价因素, 但主要是对学生学习效果的评价和教师教学工作过 程的评价。

教学评价的作用有如下几方面：(1) 对教学效果进行评价, 可以了解教学各方面的情况, 从 而判断它的质量和水平、成效和缺陷。(2) 评价对教师和学生具有监督和强化作用。(3) 利于 促进教学目标的实现, 全面提高教学质量[3]。

教学过程中对教学质量产生影响的因素是动态的, 不是一成不变的。所以对待教学评估数据, 应该用动态的眼观看待这些因素。因学生在评分的过程中的不确定性和多变性，外加一些个 人主观的想法，学生的评分无法客观公正的反映真实的教学成果 [4-5]。这种带有随机因素的 评价方法只能在一定程度上得到局部的最好评价结果, 却无法对评估数据进行公平透彻的分 析, 无法给教学评估工作提供强有力的数据支持。如何在现实教学中表达、分析和使用教学 
评估结果, 利用一个真实可行的结果调动广大教师和学生的积极性, 让教学评教数据发挥出 更大的作用, 这是目前面临的一个值得研究的课题。

随着信息技术的发展, 我们已经进入了大数据时代, 数据挖掘(Data Mining)技术的应用领域 将更加广泛。它可以利用各个行业积累的行业数据发现其内部隐藏的规律, 因此把数据挖掘 技术运用到教学质量评教中, 对评教的相关数据进行挖掘, 发现其中有价值的信息知识, 辅 助教育工作者进行决策, 全面了解学生和教师的特点和需要, 对学生学习成绩的提高和教师 教学水平的改善都很有意义[6], 这样不仅可以为教师和教务工作者指明以后努力的方向, 也 可以充分的减少教师和教务工作者的工作量。利用教学评估数据找出有利于提高教学质量的 关键因素, 找出教师个人相关信息对评教指标的限制, 教师可以把自身存在的不足及时改正, 把存在的优点积极发扬, 把最棒的教学结合到自己的教学工作中, 积极丰富教学内容、改进 教学方法, 提高教学质量。

\section{2. 评教指标体系的构建方法}

教学评价数据是对评教各分项指标分数的加权求和, 分项指标评分的确定是分项总分和指标 权重的乘积, 所以教学质量评价指标权重对评教总分有着质的影响。客观公正的评教分数对 日后的数据挖掘工作有着重要的作用, 若出现评价分数不合理则会导致挖掘分析的结果不正 确或者出现偏差, 进而会严重影响数据分析后的决策建议, 使得教学工作出现很大的失误。 由此可见, 教学质量评价指标权重的确定对评教数据的挖掘分析和决策意见起着至关重要的 作用, 要想得出科学的教学指导意见, 就必须合理分配评教指标权重。因此, 教学质量评价 指标权重分配是所有工作的重要前提 [7]。

目前的指标权重分配方法主要有基于主观因素的权重分配和基于客观条件的权重分配。目前 高校常用的评教指标的权重设定方法主要有: 层次分析法 [8]、主成分分析法、粗粘集理论方 法 [9]、模糊集理论方法 [10-11]、基于信息熵分析方法和语义权重分配方法 [12]等等。本文 采用主客观结合法, 来解决学校教学质量评价指标确定问题。采用组合权重分配方法 [13]主 要是为克服层次分析法的主观性和粗粘理论的客观性, 使得这两种方法在进行权重分配的时 候能够优势互补, 最终求出最优的权重值。

定义: 设 $w_{p i}$ 表示利用粗粩集理论求解的指标 $i$ 的权重值, $w_{q i}$ 表示利用层次分析法求解的指标 $i$ 的权重值, 其中, $1 \leqslant i \leqslant n, \sum_{i=1}^{n} \boldsymbol{w}_{p i}=\sum_{i=1}^{n} \boldsymbol{w}_{q i}=\mathbf{1}, n$ 是所有底层指标的个数。设组合权重为 $w_{i}$, $\sum_{i=1}^{n} \boldsymbol{w}_{i}=\mathbf{1}$, 对组合权重建立最优化模型公式为:

$\boldsymbol{L}=\min \left\{\sum_{i=1}^{n}\left[\theta *\left(\boldsymbol{w}_{i}-\boldsymbol{w}_{p i}\right)^{2}+(\mathbf{1}-\theta) *\left(\boldsymbol{w}_{i}-\boldsymbol{w}_{q i}\right)^{2}\right]\right\}, \quad 0 \leqslant \theta \leqslant 1$

根据 $L$ 在可行域上有唯一解, $\frac{\partial \boldsymbol{L}}{\partial \boldsymbol{w}_{i}}=\mathbf{0}$ 得到:

$2 * \theta\left(w_{i}-w_{p i}\right)+2 *(1-\theta)\left(w_{i}-w_{q i}\right)=0 \rightarrow \theta\left(w_{i}-w_{p i}\right)+(1-\theta)\left(w_{i}-w_{q i}\right)=0$

故 组合权重为:

$w_{i}=\theta^{*} w_{p i}+(1-\theta) w_{q i}, \quad 0 \leqslant \theta \leqslant 1$

\section{3. 基于权重的近邻 $K$-medoids 聚类算法}

$K$-medoids 算法是基于划分思想的一种的聚类算法[14], 它与 K-means 算法处理过程类似, 就是 在算法执行前需要对聚类数目 $K$ 和聚类中心进行初始化赋值, 之后对样本数据通过反复地迭 代计算, 最终达到使目标函数最小化的目的, 即最终能够确定的聚类中心和聚类样本不再发 生变化。两种算法最大的区别在于中心点的选取上, 这也是两个算法的核心, $K$-means 的中 
心点选取是计算当前 cluster 中所有数据样本点的平均值, $K$-medoids 是在 cluster 中选取一个 距离其它各个样本点的距离最小的样本点作为中心点, 它克服了 $K$-means 对孤立点敏感的缺 陷。PAM(partitioning around medoids)算法[15]是 $K$-medoids 算法中比较典型的一种, 尽管自身 有较强的健壮性, 但是其聚类的时间也较长, 之后的快速 $K$-medoids 算法虽然在很大程度上 降低了计算开销, 但在初始聚类中心的选取上扔有一定的缺陷, 最初的聚类中心很有可能会 选在同一个类族中, 这样在进行计算的时候就会造成很大的开销, 为此有人提出基于邻域的 快速 $K$-medoids 算法, 以便尽可能地避免初始中心落在同一类簇中。

3.1. 基于邻域的 $K$-medoids 聚类算法

概率论与数理统计是目前许多学科都开设的重要基础课, 在这两门课程中, 我们通过方差来 衡量数据和数据平均值之间的偏离程度, 方差越大说明数据分布越分散, 数据的波动性越强, 方差越小说明数据分布表现的比较集中, 数据的波动性也就越弱。如果在进行数据聚类实验 时能够对待聚类的数据选取比较集中的数据中心点作为初始聚类中心, 那么就可以在很大程 度上减少聚类的迭代次数, $K$-medoids 聚类算法的收玫速度也会在很大程度上得到改善, 因此, 选取局部方差和最小的样本作为初始聚类中心, 就能保证聚类中心位于数据波动比较小的区 域，同时避免聚类中心位于同一个类篂。

设样本数据集为 $X=\left\{X_{1}, X_{2}, \ldots, X_{n}\right\}$, 样本之间的距离公式为:

$d\left(X_{i}, X_{j}\right)=\sqrt{\sum_{k=1}^{m}\left(X_{i k}-X_{j k}\right)^{2}}$

计算每个样本 $X_{i}$ 距离其它样本的距离, 并按照升序进行排序, 选取前 Num 个样本划入到该 样本中, 计算样本的类族方差, 如下所示:

$E\left(X_{i}\right)=\frac{\sum_{j=1}^{N u m}\left[d\left(X_{i}, X_{j}\right)-\frac{\sum_{j=1}^{N u m} d\left(X_{i}, X_{j}\right)}{N u m}\right]^{2}}{N u m}$

以样本 $\mathrm{X}_{i}$ 这样的聚类中心为圆心, 标准差 $\sigma$ 为当前圆的半径, 进行邻域进一步划分, 公式如 下:

$$
\sigma=\sqrt{E\left(X_{i}\right)}
$$

$$
\text { neighbourhood }\left(X_{i}\right)=\left\{d\left(X_{i}, X_{j}\right) \leqslant \sigma, j=1,2, \ldots, n\right\}
$$

3.2. 改进的 $K$-medoids 聚类算法

基于邻域的快速 $K$-medoids 算法的核心思想是依据距离计算样本数据的波动程度, 进而进行 数据样本的归类划分, 考虑到评教数据中数据属性较多, 而且每个属性对评教数据的影响程 度也不尽相同, 本文提出一种基于权重的邻域 $K$-medoids 算法, 在计算样本属性距离时, 引 入不同的属性权重值 $W_{l}$ 。

定义给定的样本 $U=\left\{X_{1}, X_{2}, \ldots, X_{n}\right\}$, 该样本是由 $n$ 个样本数据构成, 定义属性集合 $M=\left\{m_{1}\right.$, $\left.m_{2}, \ldots, m_{n}\right\}, M$ 描述的是一个样本中有 $m$ 个属性元素, $W=\left\{w_{1}, w_{2}, \ldots, w_{m}\right\}$ 表示属性集合 $M$ 中 每个元素的权重值, 它的取值是根据信息增益率计算得出。若 $X_{i}, X_{j} \in U$, 则定义 $X_{i}$ 和 $X_{j}$ 样本 之间的距离公式为:

$$
D\left(X_{i}, X_{j}\right)=\sqrt{\sum_{l=1}^{m} w_{l}\left(X_{i l}-X_{j l}\right)^{2}}
$$

信息增益为: $\operatorname{gain}(l)=\operatorname{info}(D)-\operatorname{info}_{l}(D)$ ；信息增益率为： $\boldsymbol{W}_{\boldsymbol{l}}=\frac{\operatorname{gain}(\boldsymbol{l})}{\inf \boldsymbol{o}(\boldsymbol{D})}$

进行算法实现时引入矩阵的概念, 以样本数 $n$ 为基准构造一个 $n$ 维的空矩阵 $\mathrm{A}_{n * n} n$, 在进行样 
本之间距离计算时，选用上述公式，但需要做一个限制，其定义如下：

$D\left(X_{i}, X_{j}\right)=\left\{\begin{array}{l}\sum_{l=1}^{m} \boldsymbol{d}\left(\boldsymbol{X}_{i l}, \boldsymbol{X}_{j l}\right),(\boldsymbol{i}<\boldsymbol{j}) \\ d\left(X_{j}, X_{i}\right),(i>j)\end{array}\right.$

$(i=1, \ldots, n, j=i, \ldots, n)$

改进的聚类算法的计算过程如下:

初始化: 聚类个数 $K$, 数据样本集 $U$, 领域样本截取数 Num。

(1)上三角矩阵的构建。按照距离公式进行样本数据之间距离的计算, 在计算 $n^{2} / 2$ 个数值之后, 对对角线上的数值全部赋值为 0 。

(2)初始化聚类中心。针对矩阵每行数据, 按照从小到大的顺序依次进行排序, 截取前 Num 个 距离为最小数值的样本, 在样本内进行距离方差的计算, 对距离方差进行升序排列, 选取第 一个样本作为聚类的第一个聚类中心, 对到第一个聚类中心的距离小于第一个聚类中心标准 差的样本进行样本集删除, 之后重复第(2)步, 直到得到的聚类中心数目为 $K$ 时结束。

(3)初始化类簇。根据距离优先原则将距 $M_{i}$ 最近的样本分配给 $M_{i}$ 的类簇, 得到初始划分的类 簇分布。

(4)计算误差平方和。对现有的聚类结果进行误差平方和的计算。

(5)更新聚类中心。在每个类簇中进行聚类中心的重新选取, 依据相似性距离优先原则计算每 个聚类中心的距离和，选取当前距离和最小的样本作为新的聚类中心。

(6)更新类簇。重新依据步骤(3)进行样本的分配, 直到每个样本能够分配至最近的类簇。

(7)进行迭代运算, 直至聚类误差平方和没有变化为止。

\section{4. 实验分析}

本文针对 UCI 数据集中的 Abalone, Vehicle 和 Waveform 进行算法的验证, 将算法改进前后进 行比较, 针对本文的算法和原来的近邻 K-medoids 进行数据的聚类准确度比较结果如表 1 所示。

表 1 对比结果

\begin{tabular}{|c|c|c|c|c|c|c|c|c|}
\hline 编号 & 数据集 & 样本个数 & 属性数 & 类别数 & 算法 & $K$ & Num & 准确率 \\
\hline \multirow{2}{*}{1} & \multirow{2}{*}{ Abalone } & \multirow{2}{*}{4177} & \multirow{2}{*}{7} & \multirow{2}{*}{29} & K-medoids & \multirow{2}{*}{29} & \multirow{2}{*}{4} & $92.5 \%$ \\
\hline & & & & & 本文算法 & & & $93.4 \%$ \\
\hline \multirow{2}{*}{2} & \multirow{2}{*}{ Vehicle } & \multirow{2}{*}{946} & \multirow{2}{*}{18} & \multirow{2}{*}{4} & K-medoids & \multirow{2}{*}{4} & \multirow{2}{*}{3} & $57.3 \%$ \\
\hline & & & & & 本文算法 & & & $70.6 \%$ \\
\hline \multirow{2}{*}{3} & \multirow{2}{*}{ Waveform } & \multirow{2}{*}{5000} & \multirow{2}{*}{21} & \multirow[b]{2}{*}{ 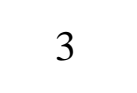 } & K-medoids & \multirow{2}{*}{3} & \multirow{2}{*}{2} & $60.9 \%$ \\
\hline & & & & & 本文算法 & & & $75.4 \%$ \\
\hline
\end{tabular}

从上述结果我们发现本文算法在不同的数据集上都表现出比原算法高的聚类准确率, 这也是 本文改进算法的初衷, 更准确的聚类效果是分析评教数据的基础。综上所述, 在同样的实验 参数前提下, 针对相同的数据集, 添加权重的算法能够得到更好的聚类准确度, 实验结果也 证明了该算法是可行的。 


\section{5. 改进的聚类算法在教评数据中的应用}

5.1. 教学评价模型

教学评价模型及相应的权值分配如表 2 所示。

表 2 教学评价权值分配表

\begin{tabular}{cccccccccccc}
\hline 一级指标 & \multicolumn{1}{c}{ 教学素质 $u_{1}(0.25)$} & \multicolumn{5}{c}{ 教学内容 $u_{2}(0.3)$} \\
\hline 二级指标 & $u_{11}$ & $u_{12}$ & $u_{13}$ & $u_{14}$ & $u_{15}$ & $u_{21}$ & $u_{22}$ & $u_{23}$ & $u_{24}$ & $u_{25}$ & $u_{26}$ \\
\hline$W$ & 0.05 & 0.05 & 0.05 & 0.05 & 0.05 & 0.05 & 0.05 & 0.05 & 0.05 & 0.05 & 0.05 \\
\hline 一级指标 & \multicolumn{1}{c}{ 教学方法 $u_{3}(0.3)$} & & & $\begin{array}{c}\text { 教学管理 } \\
u_{4}(0.1)\end{array}$ & $\begin{array}{c}\text { 教学效果 } \\
u_{5}(0.05)\end{array}$ \\
\hline 二级指标 & $u_{31}$ & $u_{32}$ & $u_{33}$ & $u_{34}$ & $u_{35}$ & $u_{36}$ & $u_{41}$ & $u_{42}$ & $u_{51}$ \\
\hline$W$ & 0.05 & 0.05 & 0.05 & 0.05 & 0.05 & 0.05 & 0.05 & 0.05 & 0.05 \\
\hline
\end{tabular}

该表由五个一级指标组成, 包括 “教学素质”、“教学内容”、“教学方法”、“教学效果” 和 “教学管理” , 这个表格中一共涉及到了 20 个相同权重的二级指标。其中各一级指标组成 如下:

(1) “教学素质”由“教态自然, 精神饱满, 和蔼可亲”、“板书美观、规范, 设计合理, 条理清晰”、 “遵守教学纪律, 责任感强”、“普通话清晰, 响亮语言流畅、精炼、生动、感染力强”、“组织 和调控课堂能力强”这五个二级指标构成。

(2) “教学内容”由“教学目的明确，时间安排合理”、“讲解简明扼要，重点突出，示范操作规范”、 “认真实践教学计划, 使其符合教学大纲要求”、“将课堂知识与课外知相结合并延伸”、“使各 个学科之间能够紧密相连”、“传授知识的过程中注重思想素质和能力的培养”六个二级指标构 成。

(3) “教学方法”由“合理有效运用现代教育技术手段”、“采用启发式教学, 讲解深入浅出”、“突 出重点, 讲清难点”、“激发学生学习兴趣和主动性”、“课堂气氛活跃, 师生活动性好”、“注 重学生动手能力、创新思维和科学作风的培养”六个二级指标构成。

(4)“教学管理”的评价指标由“教学管理”和“关注学生考勤, 课堂管理严格”两个二级指标构成。 (5)“教学效果”由“学生课堂表现”和“学生技能的提高和素质的拓展”结合起来作为主要的评价 指标。

以上教学评价模型中对五个一级指标下的 20 个二级指标，全部按照相同的权重(0.05)分配设 置, 由于各二级指标对所在的一级指标影响程度有差异, 所以这样设置并不合适, 因此我们 尝试对教学评价模型进行调整并改进, 以求能够得到更好的聚类效果。按照组合权重调整方 法-公式(2), 得到调整后的教学评价权值分配表如表 3 所示。

表 3 调整后的教学评价权值分配表

\begin{tabular}{cccccccccccc}
\hline 一级指标 & \multicolumn{1}{c}{ 教学素质 $u_{1}(0.25)$} & \multicolumn{6}{c}{ 教学内容 $u_{2}(0.3)$} \\
\hline 二级指标 & $u_{11}$ & $u_{12}$ & $u_{13}$ & $u_{14}$ & $u_{15}$ & $u_{21}$ & $u_{22}$ & $u_{23}$ & $u_{24}$ & $u_{25}$ & $u_{26}$ \\
\hline$W$ & 0.03 & 0.03 & 0.06 & 0.09 & 0.04 & 0.03 & 0.04 & 0.03 & 0.07 & 0.05 & 0.08 \\
\hline 一级指标 & \multicolumn{1}{c}{ 教学方法 $u_{3}(0.3)$} & & & $\begin{array}{c}\text { 教学管理 } \\
u_{4}(0.1)\end{array}$ & $\begin{array}{c}\text { 教学效果 } \\
u_{5}(0.05)\end{array}$ \\
\hline 二级指标 & $u_{31}$ & $u_{32}$ & $u_{33}$ & $u_{34}$ & $u_{35}$ & $u_{36}$ & $u_{41}$ & $u_{42}$ & $u_{51}$ \\
\hline$W$ & 0.04 & 0.06 & 0.03 & 0.05 & 0.09 & 0.03 & 0.06 & 0.04 & 0.05 \\
\hline
\end{tabular}

5.2. 教评数据的聚类处理

对 “计算机程序设计” 课程 300 张教评调查表样本数据, 分为 3 个聚类数目, 这三个聚类数 目分别代表 “满意”，“基本满意”，“不满意”三个等级。通过使用本文提出的改进的 
K-medoids 聚类算法进行聚类, 聚类结果情况如表 4 所示。

表 4 评教数据聚类结果

\begin{tabular}{lll}
\hline \multicolumn{1}{c}{ 簇 } & \multicolumn{1}{c}{ 样本个数 } & \multicolumn{1}{c}{ 比例 } \\
\hline 簇 1(满意) & 84 & $84 / 300=28 \%$ \\
\hline 簇 2(基本满意) & 180 & $180 / 300=60 \%$ \\
\hline 簇 3(不满意) & 36 & $36 / 300=12 \%$ \\
\hline
\end{tabular}

接着, 又收集、整理出 300 份该门课成绩数据样本，数据从 0 分到 100 分。为了方便进行比 较和记录, 我们就把成绩数据样本划分为 80 分以上、 80 分-60 分 (包括 80 分和 60 分) 以及 60 分以下三个等级。比较并分析三个数段，各分数段的比例如表 5 所示。

表 5 学生成绩分布情况表

\begin{tabular}{lll}
\hline \multicolumn{1}{c}{ 分数段 } & \multicolumn{1}{c}{ 样本个数 } & \multicolumn{1}{c}{ 比例 } \\
\hline 80 分以上 & 90 & $90 / 300=30 \%$ \\
\hline $80-60$ 分 & 180 & $180 / 300=60$ \\
\hline 60 分以下 & 30 & $30 / 300=10 \%$ \\
\hline
\end{tabular}

通过比较表 4 和表 5 发现，聚类各等级所占的比例与各分数段所占比例达到了基本一致的结 果, 这充分说明了改进后的 $K$-medoids 算法是可行的, 教师教学水平与学生学习成绩间的密 切关系得到了良好的体现和反映。这对教学管理工作产生了较好的指导意义和参考价值。

\section{6. 结论}

本文提出一种新的聚类算法-基于权重的邻域 $K$-medoids 算法, 算法充分考虑数据属性对聚类 结果影响程度不同, 引入属性权值进行样本数据的距离计算, 对邻域 $K$-medoids 算法进行改 进。针对当前评教指标权重都是依据教务部门的教学经验设定, 存在人为因素过大, 给数据 本身的统计结果造成了很大的影响问题，提出基于主客观权重相结合的方法，并把该方法用 于评教指标体系的构建。利用改进后的聚类算法对评教数据进行聚类分析, 得出数据中潜在 的一些评教信息。

本文分析的数据量和范围相对狭窄, 后期需要根据相关部门要求进行其它数据的分析, 进一 步完善评教数据分析工作。本文涉及的评教指标相对简单, 随着评教数据的增加和分析要求 的深入, 迫切需要对评教指标进行进一步的完善。

\section{7. 鸣谢}

本文得到国家自然科学基金项目(No.61303004); 国家社会科学基金重大项目(No.13\&ZD148); 福建省自然科学基金项目(No.2013J05099)的资助支持。 


\section{References}

[1] Xie anbang, Han yingxiong, Xun yuan, Luo xiaocheng, Wang daohong. Investigation and Analysis on the teaching quality after enrollment expansion of universities [J]. Research on the development of Education, 2005(8): 84-89.

[2] An min. Quantitative analysis of research literature on the evaluation of classroom teaching quality in Universities [J]. Teaching research, 2008(2): 114-116.

[3] Qiu wenjiao. Discussion on the construction of teaching culture quality [J]. Education Review, 2007(5): 17-18.

[4] Li jing. An empirical analysis on the influencing factors of the teaching quality evaluation in universities [J]. Educational and scientific research in Jiangxi, 2007(7): 62-64.

[5] Zhang cong. Comfirming study on incomparability of evaluation results for classroom teaching quality [J]. Research on Higher Engineering Education, 2003(3): 29-32.

[6] Chalaris M. Improving Quality of Educational Processes Providing New Knowledge Using Data Mining Techniques [J]. Procedia-Social and Behavioral Sciences, 2014, 147(0): 390-397.

[7] Jian ming. Research on the evaluation model of classroom teaching quality in universities[C]. Proceedings of academic annual conference 2001 for china quality association and the second china-us symposium on quality management, 2001.

[8] Wang xiuming, chen mingrui. Application of ahp hierarchy analysis approach in the comprehensive evaluation system of university teaching staff [J]. Journal of Hainan University (natural science edition), 2012(3): 277-281.

[9] YI Y, Dong Z. Neural Decoding based on Rough Set Theory [J]. International Journal of Advancements in Computing Technology, 2013, 5(9): 359-365.

[10] Weng yu. Teaching quality evaluation method based on fuzzy comprehensive evaluation [J]. Information Systems Engineering, 2011(1): 92-93.

[11]Chen J, Hsieh H, Do Q. Evaluating teaching performance based on fuzzy AHP and comprehensive evaluation approach [J]. Applied Soft Computing, 2015, 28(0): 100-108.

[12] Ohnishi S, Yamanoi T. On Fuzzy Priority Weights of AHP for Double Inner Dependence Structure [J]. Procedia Computer Science, 2014, 35(0): 1003-1012.

[13] Li Y, Xu F P. Research on The Multiple Combination Weight Based on Rough Set and Clustering Analysis-The Knowledge Transfer Risk in IT Outsourcing Taken as An Example[J]. Procedia Computer Science, 2013, 17(0): 274-281.

[14] Seyed M R Z, Mehdi M, Farahnaz S. Ranked k-medoids: A fast and accurate rank-based partitioning algorithm for clustering large datasets [J]. Knowledge-Based Systems, 2013, 39(0): 133-143.

[15]Huang xiang, Cai biye, Meng ying. A clustering algorithm based on PSO\&PAM [J]. Computer engineering and Applications, 2013(04): 149-152. 